\title{
NUMERICAL STUDY ON THE FLUID-STRUCTURE INTERACTION AND AERODYNAMIC NOISE RADIATION OF A MEMBRANE AIRFOIL
}

\author{
ELENA KOLB ${ }^{1, *}$ AND MICHAEL SCHÄFER ${ }^{1}$ \\ ${ }^{1}$ Institute of Numerical Methods in Mechanical Engineering \\ Technische Universität Darmstadt \\ Dolivostraße 15, 64293 Darmstadt, Germany \\ e-mail: kolb@fnb.tu-darmstadt.de, web page: http://www.fnb.tu-darmstadt.de
}

Key words: Membrane airfoil, Fluid-structure interaction (FSI), Computational aeroacoustics, Large eddy simulation (LES)

\begin{abstract}
This paper investigates the fluid-structure interaction phenomena and their effect on the aerodynamic noise radiation observed for a membrane airfoil subjected to a turbulent flow at a fixed angle of attack $\alpha=20^{\circ}$. For this purpose, two cases with different Reynolds numbers $(\mathrm{Re}=53,100$ and $\mathrm{Re}=79,700)$ are simulated. The applied partitioned fluid-structure interaction approach uses large eddy simulations to predict the incompressible flow problem and includes geometric non-linear effects for the structural problem. Following a hybrid method based on a hydrodynamic/acoustic splitting technique, the incompressible flow field solution provides the acoustic source term for the aeroacoustic computation. The results suggest a coupling between the vortex shedding, the dynamic structural response and the radiated aerodynamic sound for this configuration.
\end{abstract}

\section{INTRODUCTION}

Flying mammals such as bats and flying squirrels possess thin compliant wings that grant them exceptional flight capabilities. The lightweight nature of such membrane wings make them especially interesting for micro air vehicle (MAV) applications with Reynolds numbers in the low to medium range $(\operatorname{Re}=50,000-100,000)$, and also the broader field of morphing wings enjoys increasing popularity. Unlike rigid airfoils, membrane airfoils passively adapt to the flow, resulting in a complex and usually unsteady fluid-structure interaction (FSI) process.

The effect of wing flexibility on the aerodynamic performance of membrane wings has been studied for example by Song et al. [1]. They reported that membrane wings show increased lift slopes and a higher maximum lift coefficient compared with their rigid counterparts. In a comprehensive experimental study on the unsteady FSI of membrane airfoils, 
Rojratsirikul et al. [2] suggested that a coupling between the membrane oscillation and the vortex shedding exists in the studied Reynolds number range $(\operatorname{Re}=53,100-106,000)$ and for high angles of attack. On the other hand, the aerodynamic sound radiation of membrane wings has received less attention so far. The relevant studies in this field mostly refer to flapping wings (see, e.g., [3]). From an engineering point of view the question of aeroacoustic properties is often crucial and therefore requires investigation.

Although many studies have demonstrated the beneficial aerodynamic performance of membrane airfoils, the associated fluid-structure interaction processes at medium to high angles of attack are complex and not fully understood. Additionally, it is unclear how the wing flexibility affects the aerodynamic sound radiation. To better understand these effects, we present coupled fluid-structure-acoustic simulations of a membrane airfoil $\left(\alpha=20^{\circ}\right)$. The geometry under consideration corresponds to the experiments of Rojratsirikul et al. [2] on membrane airfoils for MAV applications. The partitioned FSI approach uses the flow solver FASTEST [4] to predict the hydrodynamic flow field governed by the incompressible Navier-Stokes equations with large eddy simulations (LES). The finite-element solver CalculiX [5] includes geometric non-linear effects for the structural problem. The aeroacoustic problem is treated by a hybrid approach based on a hydrodynamic/acoustic splitting technique, where the acoustic field is calculated sub-

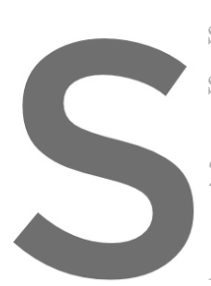
sequently to the FST

solution.

\section{2}
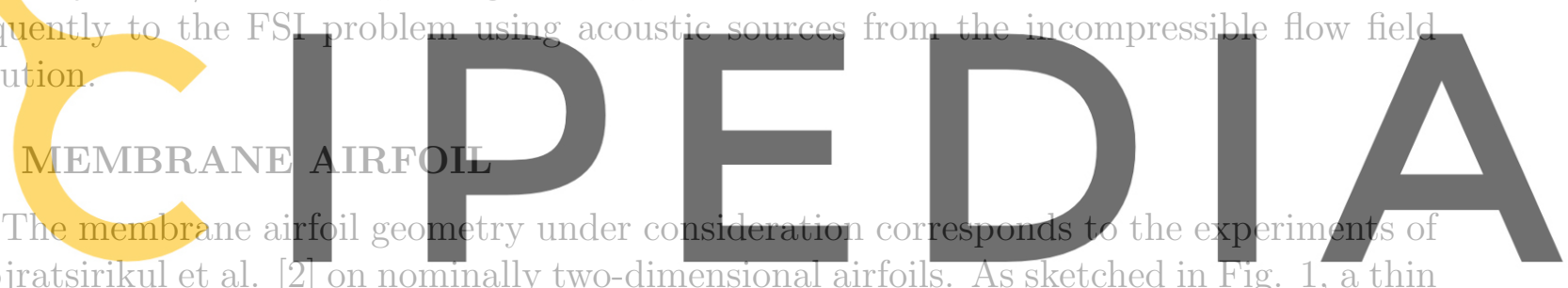

Rojratsirikul et al. [2] on nominally two-dimensional airfoils. As sketched in Fig. 1, a thin

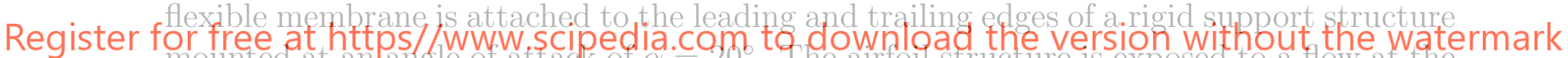
mounted at an anlgle of attack of $\alpha=20^{\circ}$. 1 he airfoll structure is exposed to a 1 low at the Reynolds number Re $=\left(c V_{\infty} \rho^{i c}\right) / \mu^{i c}$ (chord length $c=0.15 \mathrm{~m}$, density $\rho^{i c}=1.16 \mathrm{~kg} / \mathrm{m}^{3}$, dynamic viscosity $\mu^{i c}=16.415 \mu \mathrm{Pas}$ ), where $V_{\infty}$ is the undisturbed inflow velocity. Two different Reynolds numbers are investigated with $\mathrm{Re}=53,100$ and $\mathrm{Re}=79,700$. In this Reynolds number range Rojratsirikul et al. [2] measured quasi two-dimensional membrane deformations. The corresponding Mach numbers are Ma=0.022 and Ma=0.015. The material properties of the membrane with a thickness of $t=0.2 \mathrm{~mm}$ mimic the latex rubber sheet used in the reference experiment with density $\rho^{s}=1,000 \mathrm{~kg} / \mathrm{m}^{3}$ and Young's modulus $E^{s}=2.2 \mathrm{MPa}$. The experimental Poisson's ratio is not known. It is set to $\nu^{s}=0.3$ to match the DNS study of Yang et al. [6]. 


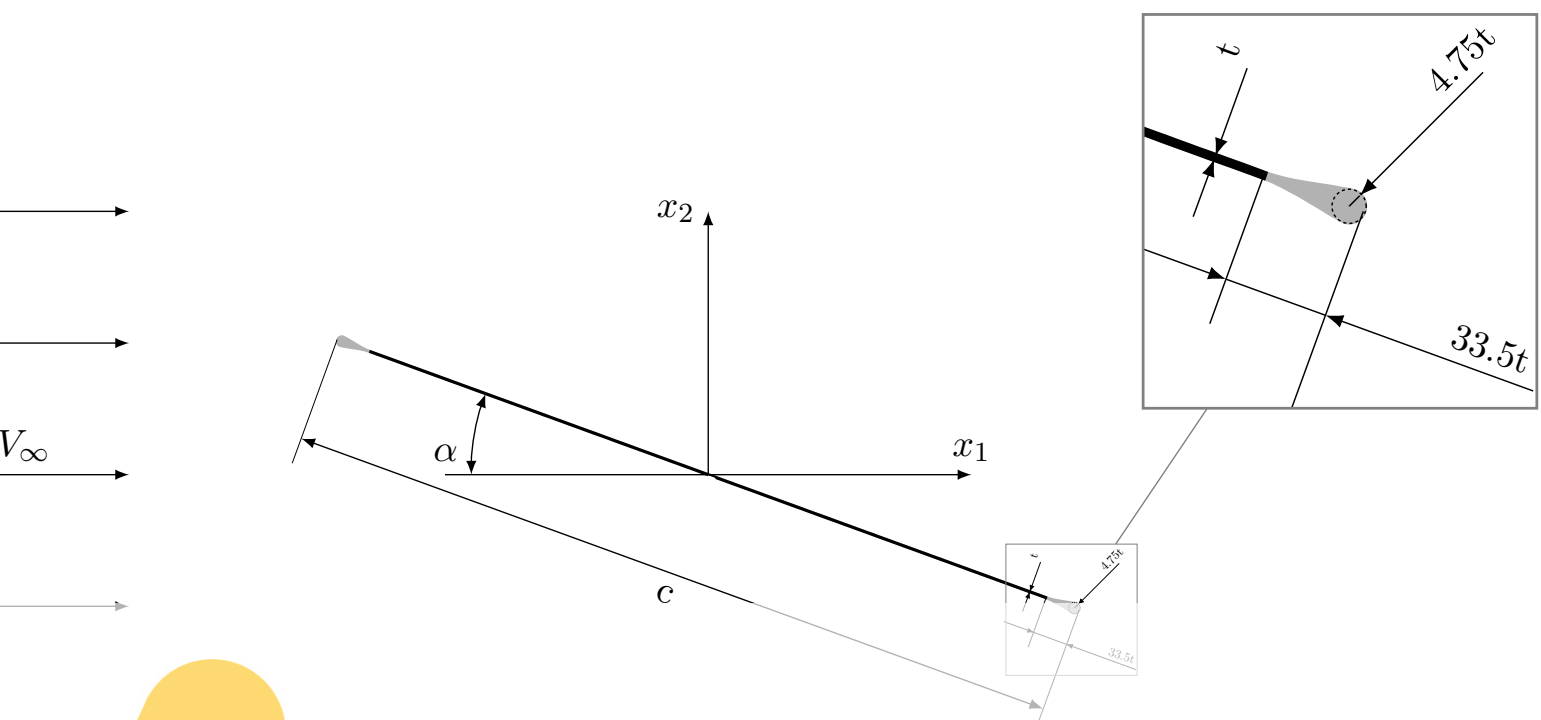

Figure 1: Sketch of the membrane airfoil configuration at an angle of attack of $\alpha=20^{\circ}$

3 NUMERICAL SIMULATION METHODOLOGY

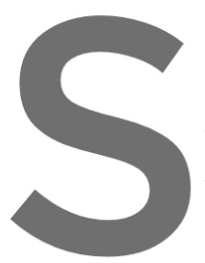

The problem domai

solid interface $\Gamma^{f s}$.

technique [7] for low

variables density $\rho$,

$\rho=\rho^{2}$
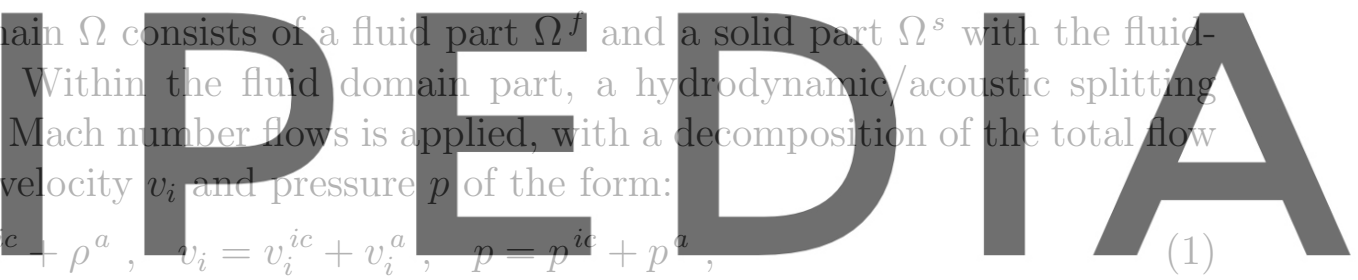

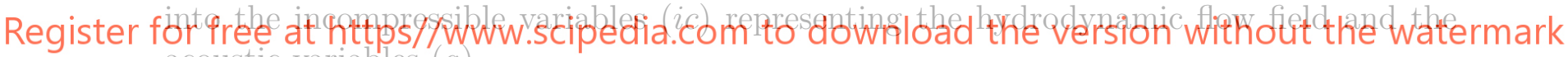
acoustic variables $(a)$.

\subsection{Computational fluid dynamics}

In the fluid domain part $\Omega^{f}$, the incompressible flow field is computed by LES using the flow solver FASTEST [4]. To incorporate moving boundaries, which arise at the fluidsolid interface $\Gamma^{f s}$, the arbitrary Lagrangian-Eulerian (ALE) approach is applied. The incompressible flow equations for a control volume $V$ with closed surface $S$ formulated in an arbitrarily moving frame of reference read:

$$
\begin{aligned}
\int_{S} v_{j}^{i c} n_{j} \mathrm{~d} S & =0, \\
\frac{\mathrm{D}}{\mathrm{D} t} \int_{V} \rho^{i c} v_{i}^{i c} \mathrm{~d} V+\int_{S} \rho^{i c} v_{i}^{i c}\left(v_{j}^{i c}-v_{j}^{g}\right) n_{j} \mathrm{~d} S & =\int_{S} \tau_{i j} n_{j} \mathrm{~d} S+\int_{V} \rho^{i c} f_{i} \mathrm{~d} V,
\end{aligned}
$$

where $v_{j}^{g}, f_{j}$ and $n_{j}$ denote the $j$-th component of the grid velocity, the external body forces and the outwards pointing unit normal vector to the surface $S$, respectively. For Newtonian fluids the stress tensor becomes: 


$$
\tau_{i j}=\mu^{f}\left(\frac{\partial v_{i}^{i c}}{\partial x_{j}}+\frac{\partial v_{j}^{i c}}{\partial x_{i}}\right)-p^{i c} \delta_{i j}
$$

where $\mu^{f}$ denotes the dynamic viscosity of the fluid and $\delta_{i j}$ denotes the Kronecker delta. In addition, the so-called space conservation law (SCL) must be fulfilled to ensure that no space is lost when a control volume changes its shape:

$$
\frac{\mathrm{D}}{\mathrm{D} t} \int_{V} \mathrm{~d} V-\int_{S} v_{j}^{g} n_{j} \mathrm{~d} S=0
$$

The flow equations are discretised in FASTEST using a finite-volume method on a blockstructured, body-fitted grid and solved using a SIMPLE type pressure correction scheme [4]. Time discretisation is performed using a second-order backward differencing scheme with a time step size of $\Delta t^{i c}=10^{-5} \mathrm{~s}$. The convective fluxes are approximated by a second-order multi-dimensional linear interpolation (MuLI) scheme in combination with a first-order upwind scheme (80\% MuLI with 20\% upwind) to stabilise the simulation. The applied subgrid-scale model is based on Germano's dynamic procedure [8]. The grid has an $\mathrm{O}$-type structure with a radius of $25 \mathrm{c}$ and spanwise extension of $L_{s}=0.6 \mathrm{c}$. The

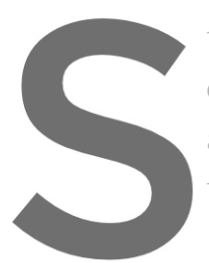
total number of contro volumes is approximately 20.3 circumferential, and applied in the spanwis the outlet.

\subsection{Computational aeroacoustics}
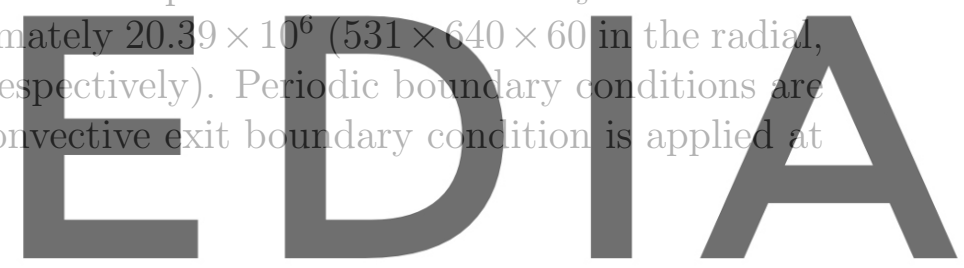

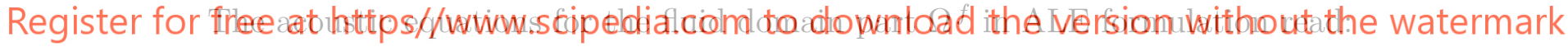

$$
\begin{aligned}
\frac{\partial \rho^{a}}{\partial t}+\rho^{i c} \frac{\partial v_{i}^{a}}{\partial x_{i}}+\left(v_{i}^{i c}-v_{i}^{g}\right) \frac{\partial \rho^{a}}{\partial x_{i}} & =0 \\
\rho^{i c} \frac{\partial v_{i}^{a}}{\partial t}+\rho^{i c}\left(v_{j}^{i c}-v_{j}^{g}\right) \frac{\partial v_{i}^{a}}{\partial x_{j}}+\frac{\partial p^{a}}{\partial x_{i}} & =0 \\
\frac{\partial p^{a}}{\partial t}+c_{\infty}^{2} \rho^{i c} \frac{\partial v_{i}^{a}}{\partial x_{i}}+\left(v_{i}^{i c}-v_{i}^{g}\right) \frac{\partial p^{a}}{\partial x_{i}} & =\underbrace{-\frac{\partial p^{i c}}{\partial t}+v_{i}^{g} \frac{\partial p^{i c}}{\partial x_{i}}}_{q},
\end{aligned}
$$

where $c_{\infty}$ is the speed of sound. Hence, the derivative of the incompressible pressure represents the major sound source term $q$. The acoustic equations are discretised and solved within the FASTEST framework using a finite-volume method. A high-resolution scheme is used based on the Osher flux limiter with $\beta=1.0$, which gives second order accuracy for smooth solutions. To comply with the multiscale character of the hydrodynamic flow field and the acoustics, a frozen fluid approach [7] is applied with $\Delta t^{i c} / \Delta t^{a}=600$. Applying periodic boundary conditions to numerically solve the acoustic equations with 
a simulated span similar to the hydrodynamic flow field can result in an unphysically correlated acoustic field $[9,10]$. Therefore, a 2D simulation is performed in the mid-span plane of the LES grid with the spanwise-averaged acoustic source

$$
\tilde{q}\left(x_{1}, x_{2}, t\right)=\frac{1}{L_{s}} \int_{-L_{s} / 2}^{L_{s} / 2} q\left(x_{1}, x_{2}, x_{3}, t\right) d x_{3} .
$$

A subsequent correction for finite bodies is not performed here, as the focus is on a qualitative assessment of the acoustic radiation. A zero-gradient boundary condition in the far-field allows the acoustic waves to leave the acoustic domain.

\subsection{Computational structural dynamics}

The structural mechanics problem, is solved using the finite-element solver Calculix [5]. The basic balance equations of momentum for the solid domain part $\Omega^{s}$ expressed in terms of the structural displacement $u_{i}^{s}$ read:

$$
\rho^{s} \frac{D^{2} u_{i}^{s}}{D t^{2}}-\frac{\partial\left(S_{j k} F_{i k}\right)}{\partial X_{j}}=\rho^{s} f_{i}
$$

with $\rho^{s}$ the structural density, $f_{i}$ the external force acting on the solid and $F_{i}$ deformation gradient large deformations and strain tensor $E_{i j}$ and
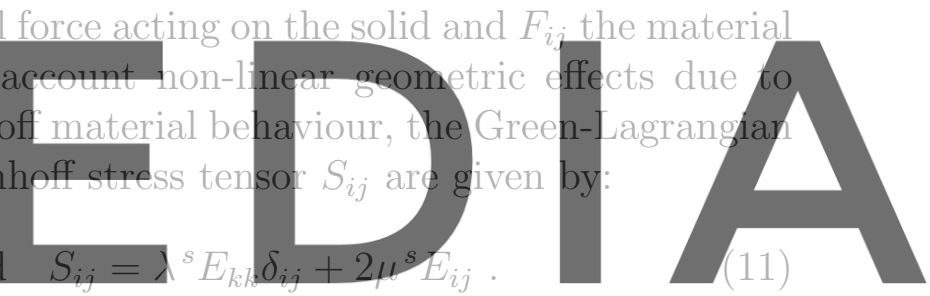

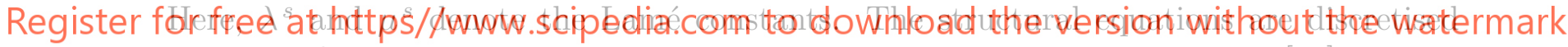
using the finite-element method and the $\alpha$-method time integration scheme [11] with an integration parameter $\alpha=-1 / 3$. The time step size is the same as for the incompressible flow problem. Motivated by the fact that the measured membrane deformations are twodimensional, two setups are investigated to reduce the computation costs of the coupled FSI simulation. First, a subset of the elastic structure is resolved with $46 \times 30$ four-node membrane elements and periodic boundary conditions. Second, the cross-section of the structure is resolved with $68 \times 2$ four-node plane strain elements with reduced integration, representing a membrane wing of infinite length. In both cases, the expansion in the spanwise direction is identical to the expansion of the flow field. The membrane is fully fixed to the support structure.

\subsection{Field coupling}

The coupling between the fluid and the solid requires the exchange of interface data, for which the coupling library preCICE [12] is used. Since the density of liquid and solid differ considerably, it is sufficient to exchange the interface data only once per time step, 
resulting in an explicit coupling scheme. On the fluid-solid interface $\Gamma^{f s}$ the following interface conditions must be fulfilled:

$$
u_{i}^{i c}=u_{i}^{a}=u_{i}^{s}, \quad \tau_{i j}^{s} n_{j}=\tau_{i j}^{i c} n_{j}, \quad \frac{\partial u_{i}^{i c}}{\partial t}=\frac{\partial u_{i}^{s}}{\partial t}, \quad \frac{\partial u_{i}^{a}}{\partial t} n_{i}=0 \quad \text { on } \quad \Gamma^{f s} .
$$

Here, $u_{i}^{i c}$ and $u_{i}^{a}$ denote the displacement of the hydrodynamic and the acoustic field, respectively. The applied splitting approach assumes that the acoustic field is considerably less energetic than the incompressible flow field for low Mach number flows. Therefore, its influence on the structure can be neglected and the acoustic field can be computed subsequently to the FSI problem. Between the incompressible flow field and the acoustic field a unidirectional coupling exists through the acoustic source term and the interface velocity is fully impressed on the incompressible flow field. Since the fluid and structural grid do not match at the interface, preCICE performs an interpolation of the exchanged data based on a radial basis function mapping with thin plate splines.

\section{RESULTS AND DISCUSSION}

The flow over a flexible membrane airfoil induces a mean camber to the airfoil and a dynamic membrane oscillation. As stated by Rojratsirikul et al. [2] and illustrated in Fig. 2 ( Re $\left.=53,100, \alpha=20^{\circ}\right)$, the roll-up
medium to high angles of attack. In the follow
membrane behaviour are presented. followed
and aeroacoustic results. In general numer
oscillation cycles after a quasi-periodic oscil
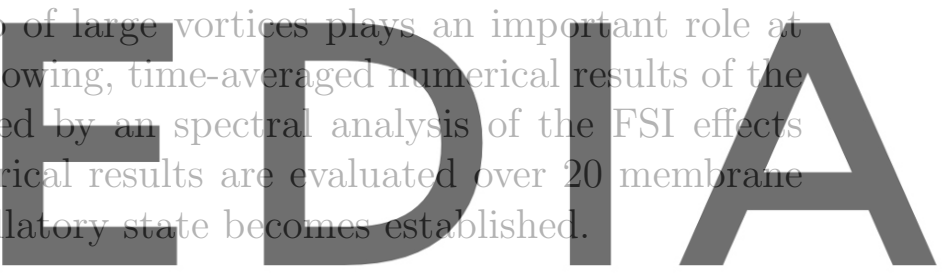

Register for free at https//www.scipedia.com to download the version without the watermark
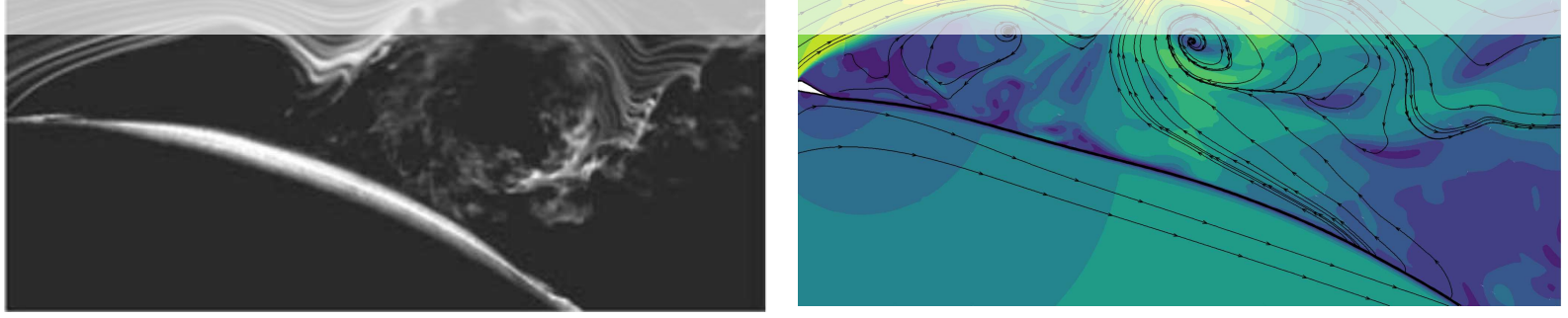

Figure 2: Visualisation of large vortice structure for $\mathrm{Re}=53,100$ using a smoke visualisation technique (image from Rojratsirikul et al. [2] (mirrored)) and a velocity magnitude and streamline plot from present LES

\subsection{Fluid-structure interaction}

Figure 3a compares the time-averaged membrane shape for $\operatorname{Re}=79,700$ to those observed in the experiment and in the simulation by Yang et al. [6]. The qualitive shape 
matches well, but in general the numerical results underpredict the maximum membrane displacement. The standard deviation of the membrane displacement in Fig. 3b indicates that the membrane oscillation exhibits a second mode at this Reynolds number. To assess the influence of the finite-element type, the mean membrane shape and the standard deviation computed with membrane elements and plane strain elements for $\operatorname{Re}=53,100$ are compared in Fig. 4. No significant difference between the two types of elements is evident. The second mode of membrane oscillation is also captured for the smaller Reynolds number in accordance with the experimental results. The maximum membrane displacement is underestimated here by $10.9 \%$ compared to the experiment, while the chordwise location of the maximum membrane displacement deviates by only $2.1 \%$. In summary, the maximum membrane displacement is underpredicted by the simulations, while the qualitative mean shape and dynamic response compare well with the experiment.
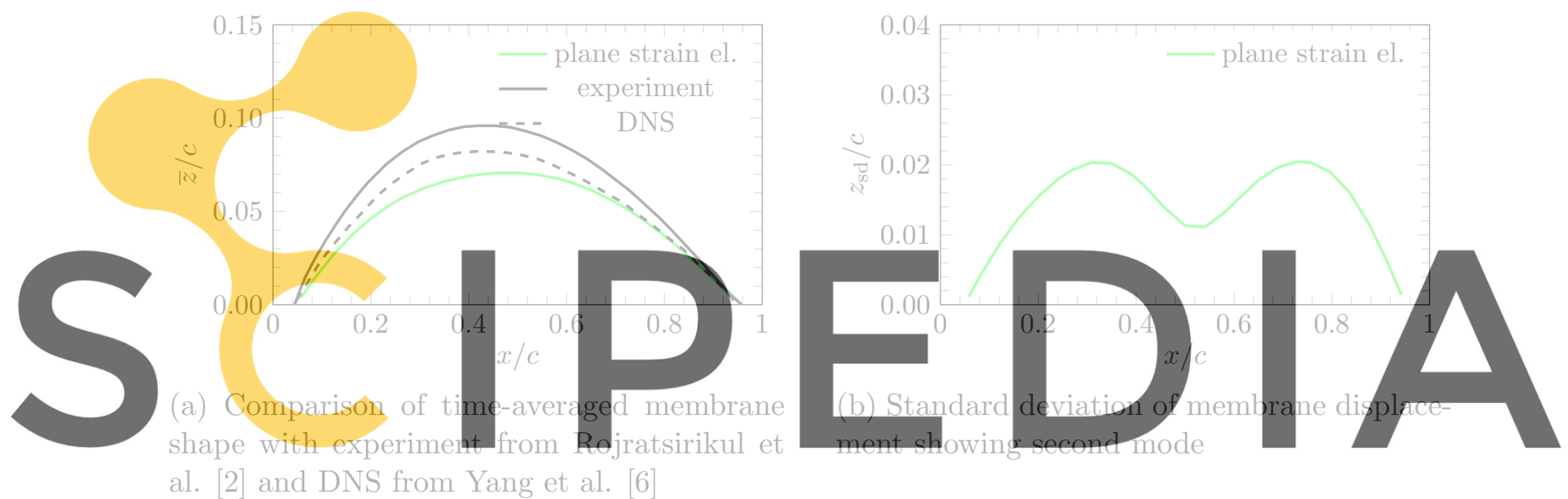

Register for free at https//www.scipedia.com to download the version without the watermark Figure 3: Mean membrane shape and oscillation mode for $\mathrm{Re}=79,700$

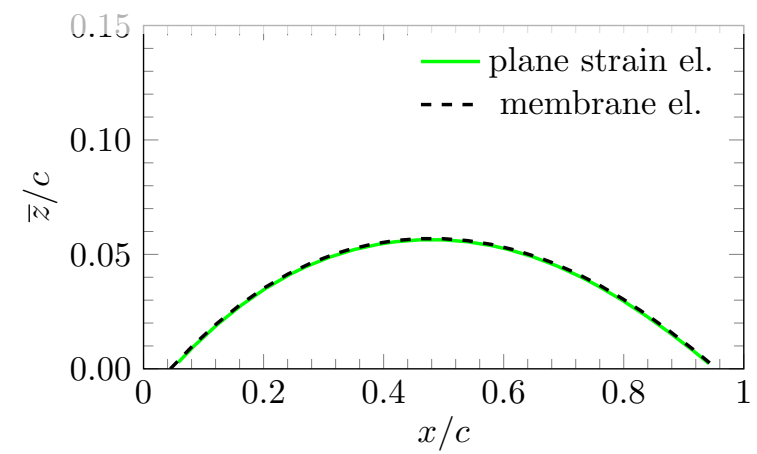

(a) Comparison of time-averaged membrane shape for membrane and plane strain elements

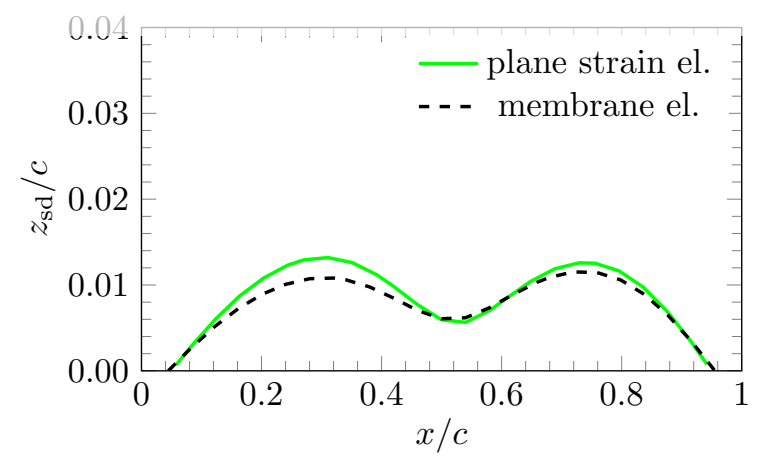

(b) Standard deviation of membrane displacement for membrane and plane strain elements showing second mode

Figure 4: Mean membrane shape and oscillation mode for $\operatorname{Re}=53,100$ 
A spectral analysis is performed using the Fast Fourier Transform (FFT) algorithm in MATLAB. Figure 5 presents the amplitude spectrum of the membrane deformation measured at three different chordwise locations. The amplitude spectrum of the membrane deformation at $x=0.25 c$ and $x=0.75 c$ each shows a single dominant frequency, where the Strouhal number is close to unity. On the other hand, the amplitude spectrum calculated from the lift coefficient presented in Fig. 6 reveals several main frequencies in the flow field. The first peak around $S t=(f c) / V_{\infty} \approx 0.5$ is associated with the natural vortex shedding frequency for thin airfoils and flat plates. The second major frequency $S t \approx 1$ coincides with the membrane oscillation frequency. A third peak around twice the membrane oscillation frequency $S t \approx 2$ is more pronounced for the smaller Reynolds number. The spectral analysis supports the observation by Rojratsirikul et al. [2] that a coupling exists between the vortex shedding and the membrane oscillation.
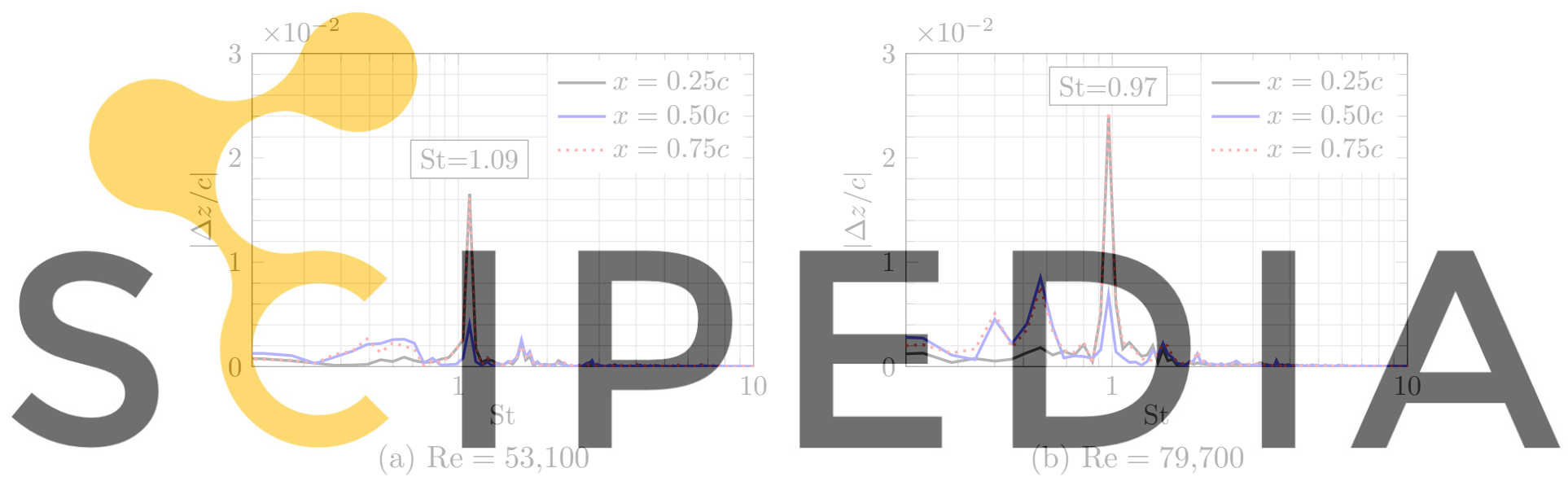

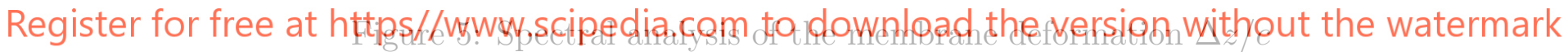

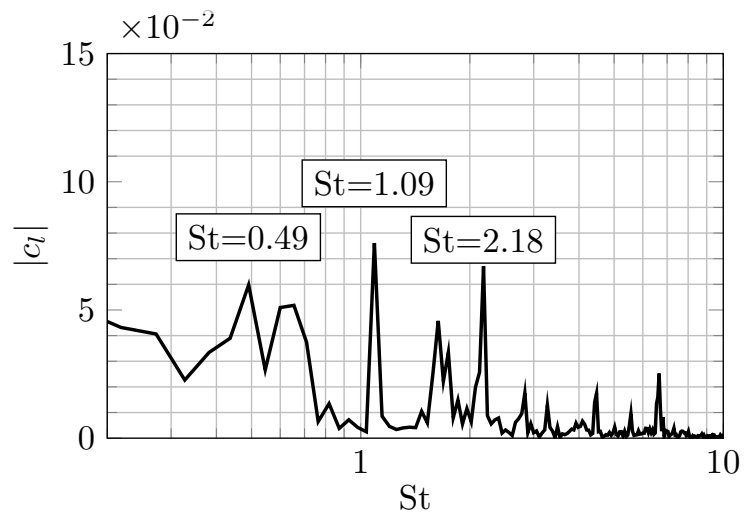

(a) $\operatorname{Re}=53,100$

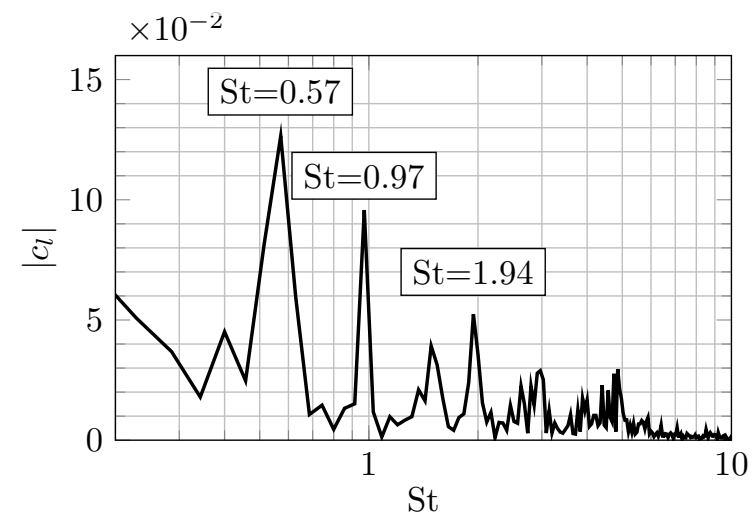

(b) $\operatorname{Re}=79,700$

Figure 6: Spectral analysis of the lift coefficient $c_{l}$ 


\subsection{Aeroacoustic results}

The aeroacoustic computation using the hydrodynamic/acoustic splitting approach is based on the acoustic source term coming from the incompressible flow field. Figure 8 illustrates the time evolution of the computed acoustic pressure field and the associated acoustic source term distribution over one cycle of the membrane oscillation for $\mathrm{Re}=79,700$. The results indicate a dipole sound generation at double the membrane's oscillation frequency $(t / T=0$ and $t / T=2 / 4)$ due to the second mode oscillation. Additionally, the source term distribution shows a mix of low-frequency components due to the shedding of large-scale coherent vortices and high-frequency small-scale turbulent structures at the trailing and leading edge. The sound pressure level at a monitor point $R=10 c$ above the membrane airfoil $\left(\Theta=90^{\circ}\right)$ presented in Fig. 7 confirms the dominant frequency at a Strouhal number around two, which corresponds to twice the membrane oscillation frequency.
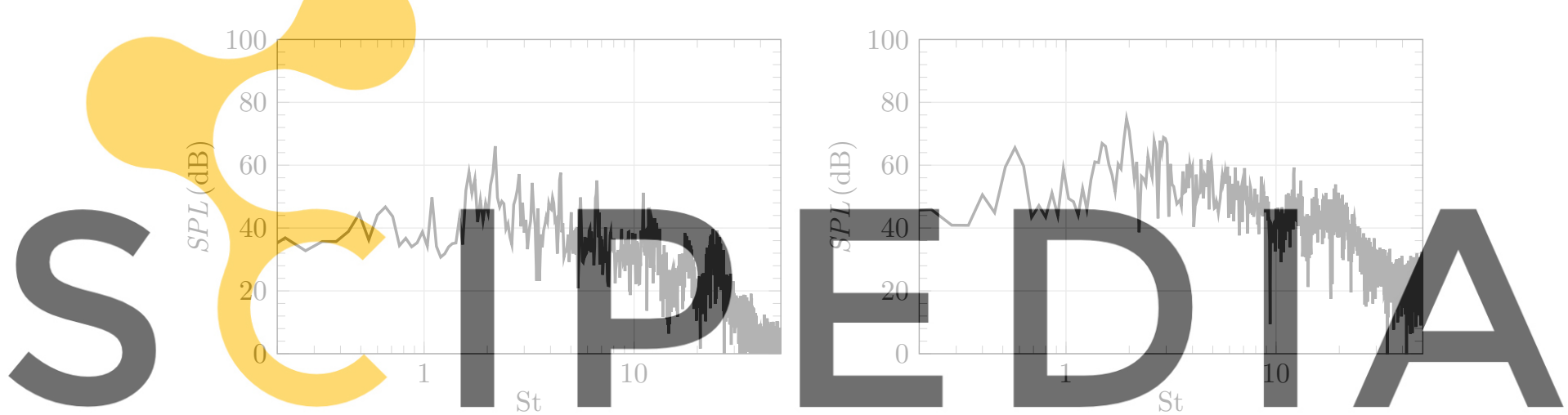

(a) $\mathrm{Re}=53,100$

(b) $\operatorname{Re}=79,700$

Register for free at https//www.scipedia.com to download the version without the watermark

Figure 7: Sound pressure level (SPL) at $R=10 c, \Theta=90^{\circ}$

\section{CONCLUSIONS}

This study investigates the FSI and aeroacoustic characteristics of a membrane airfoil subjected to a turbulent flow and mounted at $\alpha=20^{\circ}$. The qualitative membrane shape and the membrane dynamics agree well with the experimental reference data even though the maximum membrane deformation is underpredicted. A spectral analysis provides further evidence for a coupling between the vortex shedding and the dynamic structural response of the membrane. The acoustic results show a characteristic frequency at twice the membrane oscillation frequency and therefore suggest a coupling between the sound radiation and the dynamic structural response.

\section{ACKNOWLEDGEMENT}

Calculations for this research were conducted on the Lichtenberg high performance computer of the TU Darmstadt. 

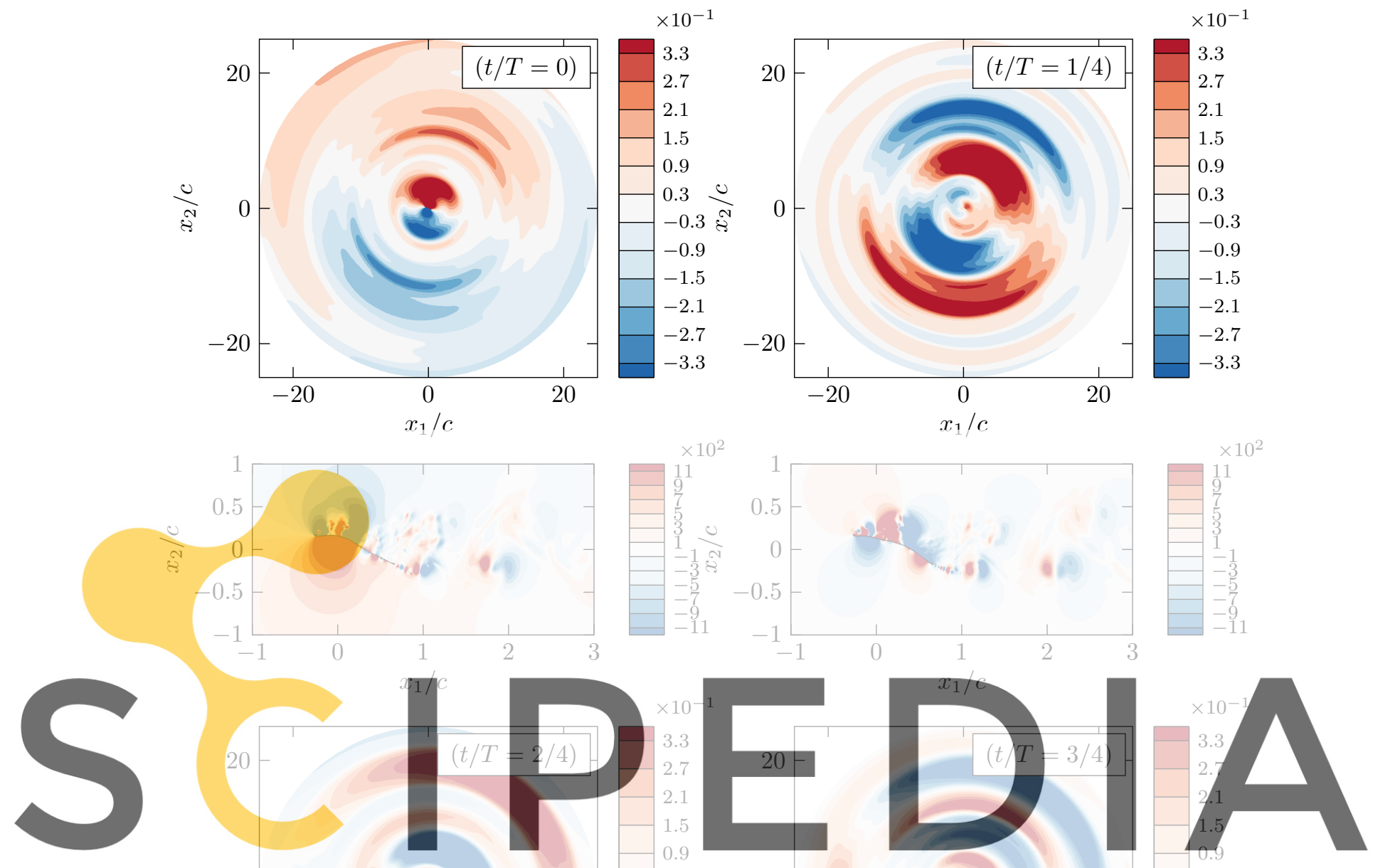

Register for
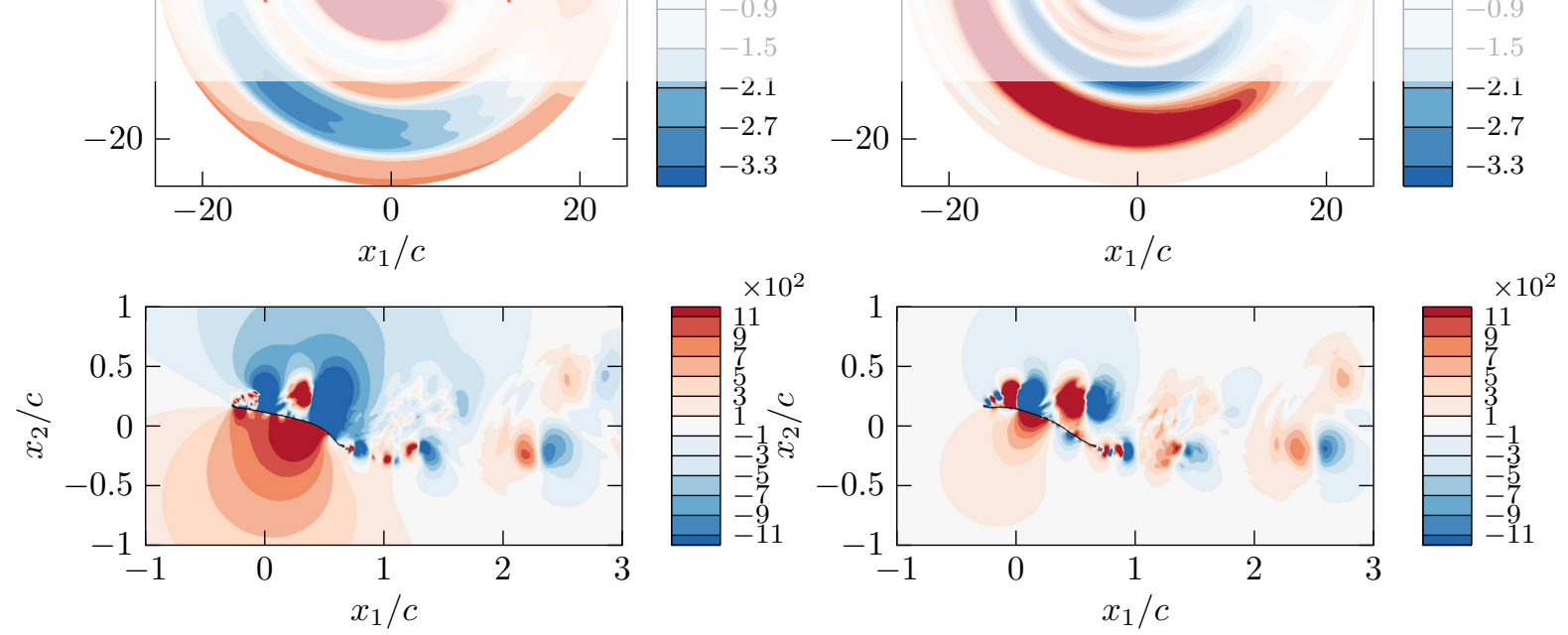

Figure 8: Visualisation of the two-dimensional acoustic pressure field $\tilde{p}^{a}$ (in $\mathrm{Pa}$ ) and the acoustic source term $\tilde{q}$ (in $\mathrm{Pa} / \mathrm{s}$ ) for $\mathrm{Re}=79,700$ 


\section{REFERENCES}

[1] Song, A., Tian, X., Israeli, E., Galvao, R., Bishop, K., Swartz, S. and Breuer, K. Aeromechanics of Membrane Wings with Implications for Animal Flight. AIAA Journal (2008) 46(8):2096-2106.

[2] Rojratsirikul, P., Wang, Z. and Gursul, I. Unsteady fluid-structure interactions of membrane airfoils at low Reynolds numbers. Experiments in Fluids (2009) 46:859872 .

[3] Geng, B., Xue, Q., Zheng, X., Liu, G., Ren, Y., and Dong, H. The effect of wing flexibility on sound generation of flapping wings. Bioinspiration 8 Biomimetics (2017) 13(1):016010.

[4] Technische Universität Darmstadt, Institute of Numerical Methods in Mechanical Engineering, Darmstadt. FASTEST Manual, (2005).

[5] Dhondt, G. and Klaus, W. CalculiX: A Three-Dimensional Structural Finite Element Program. http://www.calculix.de (2003).

[6] Yang, H.Q., Dudley, J. and Harris, R.E. Aeroelasticity Validation Study for a ThreeDimensional Membrane Wing. AIAA Journal (2018) 56(6):2361-2371.

[7] Kornhaas, M., Schäfer, M. and Sternel, D.C. Efficient numerical simulation of aeroacoustics for low Mach number flows interacting with structures. Computational Mechanics (2015) 55(6):1143-1154.

[8] Germano, M., Piomelli, U., Moin, P. and Cabot, W.H. A dynamic subgrid-scale eddy viscosity model. Physics of Fluids A: Fluid Dynamics (1991) 3(7):1760-1765.

[9] Oberai, A.A., Roknaldin, F. and Hughes, T.J.R. Trailing-Edge Noise Due to Turbulent Flows. Technical Report, Report No. 02-002, Boston University (2002).

[10] Ewert, R. and Schröder, W. On the simulation of trailing edge noise with a hybrid LES/APE method. Journal of Sound and Vibration (2004) 270(3):509-524.

[11] Hilber, H.M., Hughes, T.J.R. and Taylor, R.L. Improved numerical dissipation for time integration algorithms in structural dynamics. Earthquake Eng. Struct. Dyn. (1977) 5(3):283-292.

[12] Bungartz, H.-J., Lindner, F., Gatzhammer, B., Mehl, M., Scheufele, K., Shukaev, A. and Uekermann, B. preCICE - A Fully Parallel Library for Multi-Physics Surface Coupling. Computers and Fluids (2016) 141:250-258. 